\title{
STUDY OF SERUM VITAMIN D LEVEL IN ISCHAEMIC HEART DISEASE - A CROSS-SECTIONAL STUDY
}

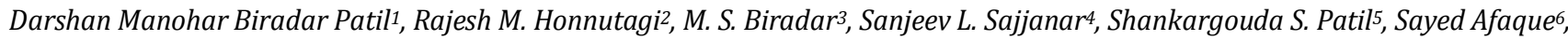
Avinash J7, G. S. Mahishale ${ }^{8}$

${ }_{1}^{1}$ Postgraduate Student, Department of General Medicine, Shri. B. M. Patil Medical College, Vijayapura, Karnataka.

2Professor, Department of General Medicine Shri. B. M. Patil Medical College, Vijayapura, Karnataka.

3 Professor, Department of General Medicine, Shri. B. M. Patil Medical College, Vijayapura, Karnataka.

${ }^{4}$ Professor, Department of Interventional Cardiology, Shri. B. M. Patil Medical College, Vijayapura, Karnataka.

${ }_{5}^{5}$ Assistant Professor, Department of General Medicine, Shri. B. M. Patil Medical College, Vijayapura, Karnataka.

${ }^{6}$ Senior Resident, Department of General Medicine, Shri. B. M. Patil Medical College, Vijayapura, Karnataka.

${ }^{7}$ Postgraduate Student, Department of General Medicine, Shri. B. M. Patil Medical College, Vijayapura, Karnataka.

${ }^{8}$ Professor, Department of General Medicine, Shri. B. M. Patil Medical College, Vijayapura, Karnataka.

\begin{tabular}{l}
\hline ABSTRACT \\
BACKGROUND \\
Vitamin D deficiency is highly prevalent worldwide, and is also noted to be high in India. Low levels of $25(\mathrm{OH}) \mathrm{D}$, the principle \\
circulating storage form of vitamin D, is present in as many as one third to one half of otherwise healthy middle aged to elderly \\
population. Endothelial dysfunction plays an important role in pathogenesis of CAD and vitamin D deficiency is postulated to \\
promote endothelial dysfunction. Because hypovitaminosis D is prevalent and easily correctable, establishing the relationship \\
between vitamin D and risk of acute coronary Syndrome is important.
\end{tabular}

Objectives- 1 . To estimate vitamin D levels in IHD patients, 2) To correlate vitamin D levels with complications of IHD.

\section{MATERIALS AND METHODS}

A Cross Sectional study of 80 patients admitted to BLDEU's Shri B. M. Patil Medical College Hospital and Research Centre, Vijayapura between December 2014 to March 2016 with diagnosis of IHD. Patients aged more than 18 years were included in studies. The Vitamin D levels were analysed in all the patients and correlated with different parameters for statistical significance.

\section{RESULTS}

During our study period 80 patients were assessed as mentioned above. The mean age of our study group was $58.5 \pm 9.6$ years. Of the study population, there were 57 male patients and 23 were females accounting to $71.2 \%$ and $28.8 \%$ respectively. Out of 80 patients 66 had Vitamin D deficiency in 45 were males and 21 were females. In that 34 patients had heart failure with hypotension and 1 had ventricular tachycardia, I had Left Ventricular Apical clot.

\section{CONCLUSION}

There is a high prevalence of Vitamin D deficiency (82.5\%) among acute coronary syndrome patients. The mean age group is $58.5 \pm 9.6$ years. Vitamin D deficiency is associated with increased risk of complications $(75.75 \%)$ in acute coronary syndromes. Vitamin D deficiency is one of the independent risk factor for acute coronary syndrome. Vitamin D deficiency is noticed more in STEMI. Vitamin D deficiency in females was more common than compared to males.

\section{KEYWORDS}

Acute Coronary Syndromes, Vitamin D deficiency, IHD (Ischemic Heart Disease), Left Ventricular Apical Clot, Ventricular Tachycardia.

HOW TO CITE THIS ARTICLE: Patil DMB, Honnutagi RM, Biradar MS, et al. Study of serum vitamin D level in ischaemic heart disease - A cross-sectional study. J. Evolution Med. Dent. Sci. 2017;6(8):624-627, DOI: 10.14260/Jemds/2017/134

\section{BACKGROUND}

Ischemic heart disease is the most common, serious, chronic life threatening illness in the world. It is a worldwide epidemic in terms of mortality. ${ }^{1}$ IHD is now occurring in low income and middle income countries. ${ }^{1}$

It is well known that common risk factors are Age, sex, Family History, Genetic factors, which are non-modifiable and modifiable risk factors are smoking, hypertension,

Financial or Other, Competing Interest: None.

Submission 20-12-2016, Peer Review 12-01-2017,

Acceptance 19-01-2017, Published 25-01-2017.

Corresponding Author:

Dr. Darshan Manohar Biradar Patil,

S/o. Dr. M. H. Biradar Patil,

Dr. Biradar's Maternity and Nursing Home,

Mudhol, Bagalkot, Karnataka.

E-mail: darshanbrpatil.dmbp@gmail.com

DOI: $10.14260 /$ jemds $/ 2017 / 134$ dyslipidaemia, diabetes mellitus, obesity, sedentary life style, stress. Still our understanding of how to prevent and treat the traditional cardiovascular risk factors is largely unknown. There is need for further investigations to know the other non-traditional risk factors. ${ }^{1}$ The common non-traditional risk factors are persons living in high altitudes, decreased sun exposure, dark skinned people.

Vitamin D deficiency is highly prevalent in the United States and worldwide. ${ }^{2}$ Although the best characterized sequelae of vitamin D deficiency involve the musculoskeletal system, a growing body of evidence suggests that low levels of vitamin D may adversely affect the cardiovascular system ${ }^{3}$. Vitamin D receptors have a broad tissue distribution that includes vascular Smooth muscle ${ }^{4,5}$ endothelium ${ }^{6}$ and cardiomyocytes. $^{7}$

Fraternity Vitamin D has been tagged as very important triggering factor for cardiovascular disease (CVD). ${ }^{8}$ Vitamin D deficiency is a highly prevalent condition. The vitamin $\mathrm{D}$ axis 
affects vascular smooth muscle cell proliferation, inflammation, vascular calcification, Renin-Angiotensin System (RAS), and blood pressure all of which affect the risk of CVD and myocardial infarction (MI). Low vitamin D level causes an increase in insulin resistance, hypertension, inflammation, and increased cardiovascular risk. Vitamin D deficiency is emerging as one more important risk factor for Ischemic Heart Disease so as the prevalence of Vitamin D as risk factor is not studied in this part of state hence study is undertaken.

\section{MATERIALS AND METHODS}

\section{Source of Data}

The information for the study will be collected from ISCHEMIC Heart Disease patients admitted to BLDEU's Shri B. M. Patil Medical College Hospital and Research Centre, Vijayapura between December 2014 to March 2016.

\section{Method of Collection of Data}

Information will be collected through prepared proforma from each patient. All patients will be interviewed as per the prepared proforma and then complete clinical examination will be done.

\section{Inclusion Criteria}

Patients with Acute coronary syndrome [Stable Angina, Unstable Angina, ST Elevation MI, Non-ST Elevation MI].

\section{Exclusion Criteria}

All patients with modifiable risk factors of IHD are excluded like Hypertension, Type 2 Diabetes Mellitus, Alcohol, Smoking.

\section{Type of Study}

Cross Sectional Study.

\section{Sample Size}

With $95 \%$ level of confidence and expected prevalence of Ischaemic heart disease as $28.5 \%$ and $\pm 10 \%$ margin of error the minimum sample size is 80 patients. $^{9}$

So the formula- N=Z2. P (1-P)/D2.

Where $\mathrm{Z}=1.96$ at $95 \%$ level of confidence.

$\mathrm{P}=$ Prevalence.

$\mathrm{D}=$ margin of error.

\section{Methods}

$5 \mathrm{ml}$ of blood is drawn from patients with IHD and serum samples sent to a centralized laboratory for analysis using the ELECYS assay. Normal 25(OH) D levels are $30 \mathrm{ng} / \mathrm{ml}$, and patients with levels $<30$ and $>20 \mathrm{ng} / \mathrm{ml}$ were classified as in sufficient and those levels with $20 \mathrm{ng} / \mathrm{ml}$ as deficient. 1

\section{Statistical Method}

The Data is analysed using Following Presentations

1. Mean \pm SD.

2. Graphical presentation

3. Chi-square test.

4. Student t-test.

\section{RESULTS}

Careful statistical analysis was performed on the obtained raw data, and following explanatory graphs were constructed for better insight of topic. In our study 80 patients were studied. The mean age of our study group was $61 \pm 12.1$ years. Of the study population, there were 57 male patients and 23 were females accounting to $71.2 \%$ and $28.8 \%$ respectively. Out of 80 patients 66 had Vitamin D deficiency in 45 were males and 21 were females. In that 34 patients had heart failure with hypotension and one had ventricular tachycardia, one had Left Ventricular Apical clot.

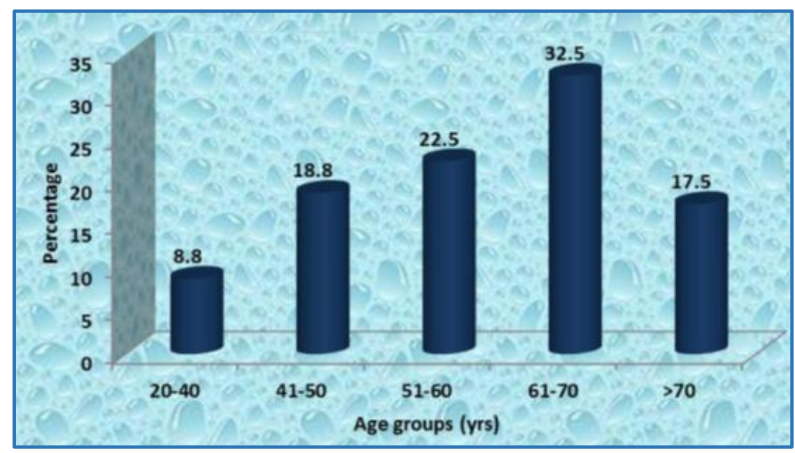

Graph 1. Distribution of cases according to age

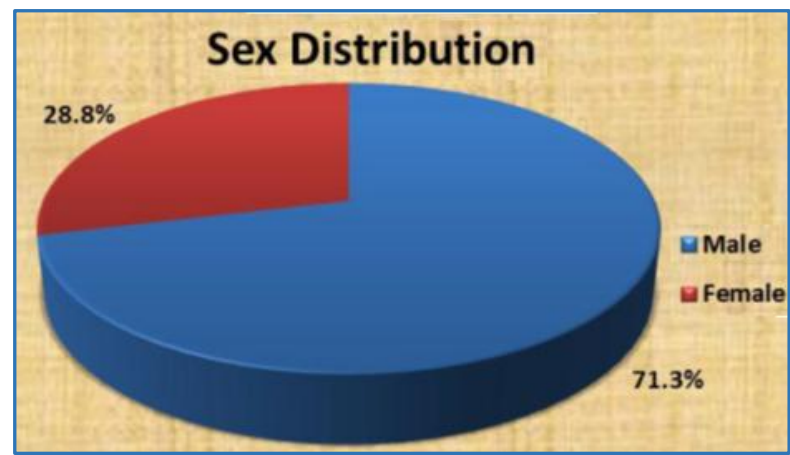

Graph 2. Distribution of cases according to Sex

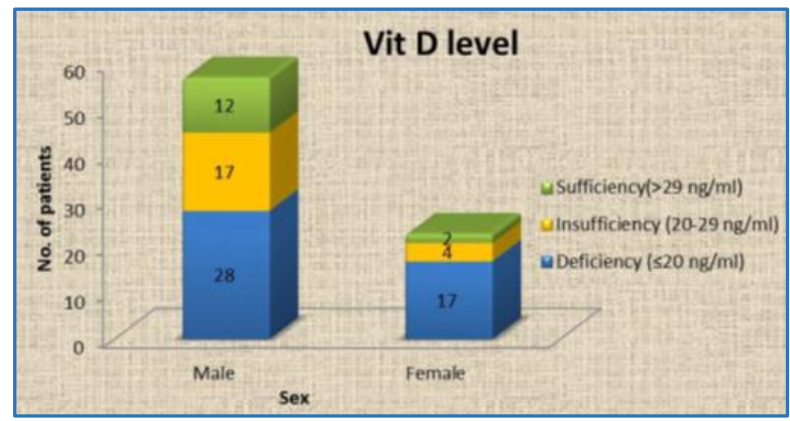

Graph 3. Distribution of cases according to vit D level

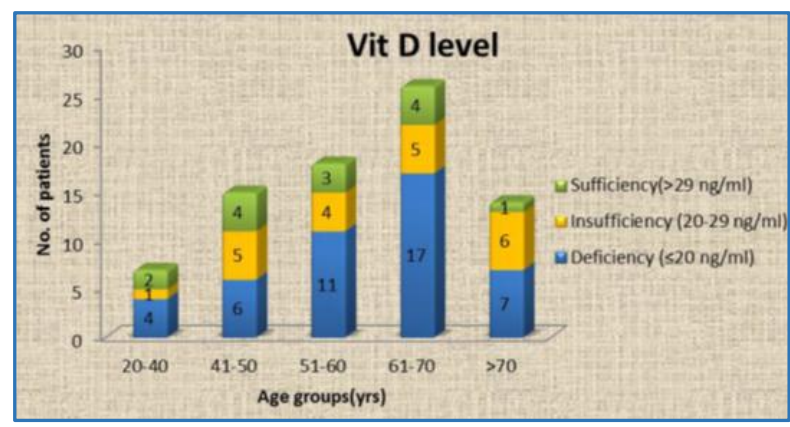

Graph 4. Distribution of cases according to age group 


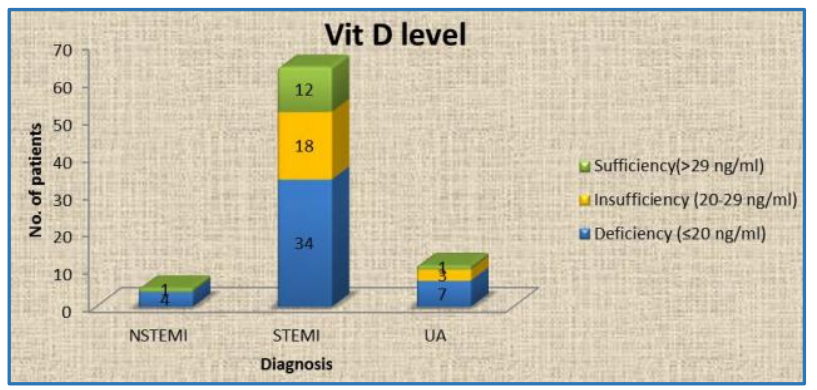

Graph 5. Distribution of cases according to ACS associated with vit D level

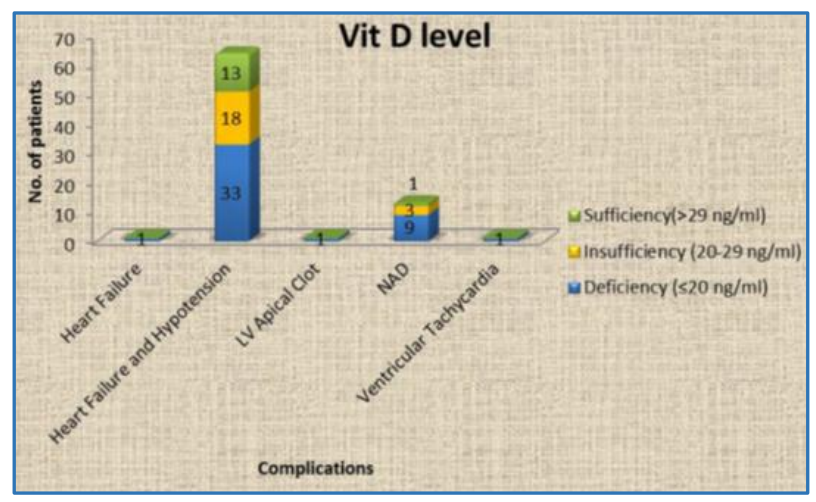

Graph 6. Distribution of cases according to complications associated with vit D level

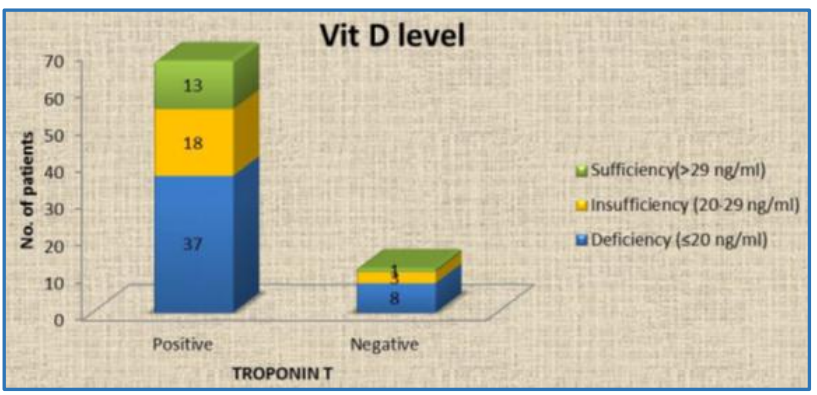

Graph 7. Distribution of cases according to troponin $T$

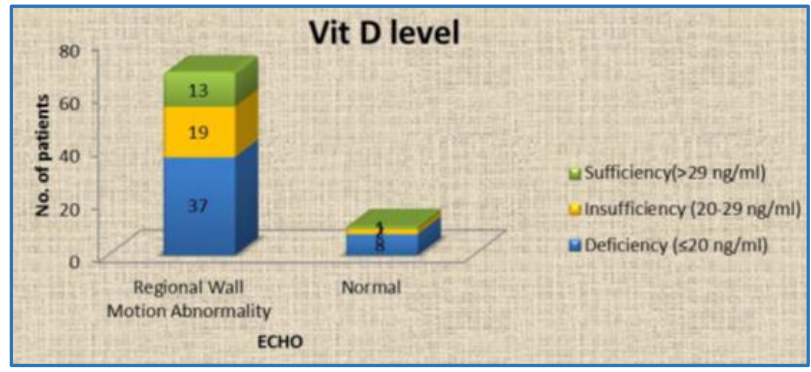

Graph 8. Distribution of cases according to ECHO findings

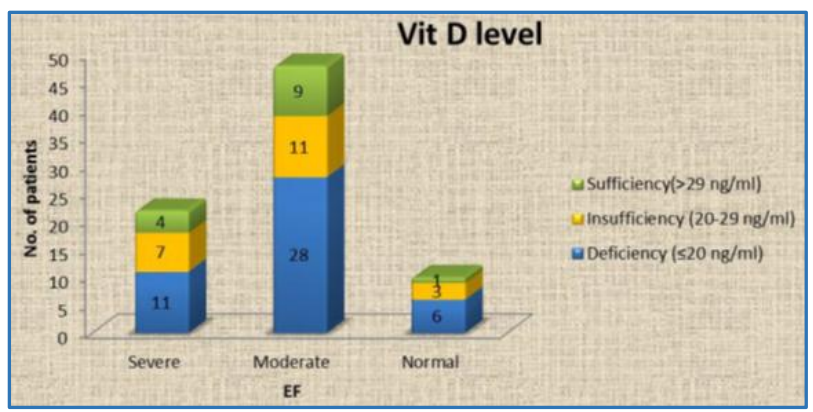

Graph 9. Distribution of cases according to EF findings

\section{DISCUSSION}

With acute coronary syndromes being one of the leading causes of mortality and morbidity all over the world, science has spread its arms in every possible direction to unsheathe the hidden reversible risk factors for ACS. Vitamin D levels have been the point of interest for many authors for a very long time.

In our study the mean age was $58.5 \pm 9.6$ years with the mean age in Vitamin D deficient patients being slightly higher 61 years, in a study from Bangalore by Karur $S$ et al the mean age was 54.09 years which goes in agreement with our study ${ }^{1}$.

The male female ratio there were more males compared to females of double the ratio male: female ratio being 2.4:1. We found a significant Vitamin $D$ deficiency in female patients compared to male patients. In our study $73.91 \%$ of females had Vitamin D deficient. The study by Karur S et al did not find any difference with respect to sex in their study ${ }^{1}$.

Our data suggested that there is high prevalence of vitamin D deficiency or insufficiency $(82.5 \%)$ in acute coronary syndrome patients. This data is consistent with a study by Satyamurthy et al ${ }^{10}$ who reported a $72 \%$ Vitamin D deficiency in AMI patients. The study by Karur $S$ et al reported $83.5 \%$ deficient or in deficient subjects in their study. A study by Scragg $\mathrm{R}$ et al also notes the inverse relationship between the vitamin $\mathrm{D}$ levels and the incidence of cardiovascular disease.

Our study also correlated with Martins, D et al ${ }^{11}$ Serum 25 $(\mathrm{OH})$ D levels are associated with important cardiovascular disease risk factors in US adults. Prospective studies to assess a direct benefit of cholecalciferol (vitamin D) supplementation on cardiovascular disease risk factors are warranted.11 Vitamin D deficiency has important cardiovascular risk factor in our study.

Our study also correlated with Pilz, S et al ${ }^{12}$ Vitamin D deficiency is common, and the cardiovascular system is a target tissue for vitamin D. Experimental studies showed beneficial vitamin $D$ effects on cardiovascular risk factors, the heart and the blood vessels. Clinical studies have largely, but not consistently, indicated that cardiovascular disease and mortality are associated with vitamin D deficiency.

In our study, there is $82.5 \%$ vitamin D deficiency in acute coronary syndrome which correlated with study done by Marijana Kneæevi Ê PraveËek et al of 60 patients with ACS, mean 25(OH)D level was $34.9 \mathrm{nmol} / \mathrm{L}$; vitamin D deficiency was present in $76 \%$ and only $8 \%$ had optimal $25(\mathrm{OH}) \mathrm{D}$ levels.

Our study also correlated with Seong Jae Hur et al ${ }^{13}$ that Lower 25D levels appear to be associated with cTnT elevation, predicting worse $\mathrm{CV}$ outcome, and are possible to involve cardiac hypertrophy or coronary artery disease.

Our study could not correlate with Mahdavi $\mathrm{K}$ et al ${ }^{14}$ in which they concluded that Significant $(\mathrm{p}<0.01)$ correlation between the vitamin D deficiency and ACS in comparison to healthy controls was recorded. As in our study the $\mathrm{p}=0.188$. Study recorded a very high vitamin $D$ deficiency in patients of ACS and suggests significant correlation between the two. Vitamin D deficiency is a worldwide health problem. A very high prevalence $(96 \%)$ of vitamin D deficiency has been reported in patients of coronary artery disease.

Our study also correlated with Giovannucci $\mathrm{E}$ et $\mathrm{al}^{3}$ that low levels of 25(OH)D are associated with higher risk of myocardial infarction in a graded manner, even after 
controlling for factors known to be associated with coronary artery disease.

Our study also correlated with Suzanne Judd et al ${ }^{15}$ that Vitamin D insufficiency is very common in the United States and world-wide and demonstrated a strong association between vitamin $\mathrm{D}$ insufficiency and risk of cardiovascular disease.

Our study also correlated with Mohammed Ahmed Abdel Rahman et al

In which he had concluded that reduced vitamin $\mathrm{D}(<20$ $\mathrm{ng} / \mathrm{ml}$ ) appears to be associated with worse systolic functions in terms of end systolic volume and end systolic dimension.

Based on this above studies correlation it could be advised to get Vitamin D levels in all patients admitted with Ischemic heart diseases so that to initiate treatment for Vitamin D deficiency in order to improve the outcome.

\section{CONCLUSION}

1. There is a high prevalence of Vitamin D deficiency $(82.5 \%)$ among acute coronary syndrome patients.

2. The mean age group is $58.5 \pm 9$.6 years.

3. Vitamin D deficiency in females was more common than compared to males.

4. Vitamin D deficiency is noticed more in STEMI.

5. Lower the Vitamin D levels more the regional wall motion abnormality.

6. Vitamin D deficiency is associated with increased risk of complications $(75.75 \%)$ in acute coronary syndromes.

7. Significant decrease in ejection fraction with deficient Vitamin D levels.

8. Vitamin D deficiency is one of the independent risk factor for acute coronary syndrome.

\section{REFERENCES}

[1] Karur S, Veerappa V, Nanjappa MC. Study of vitamin D deficiency prevalence in acute myocardial infarction. IJC Heart \& Vessels 2014;3:57-9.

[2] Lee JH, O'Keefe JH, Bell D, et al. Vitamin D deficiency an important, common and easily treatable cardiovascular risk factor? J Am Coll Cardiol 2008;52(24):1949-56.

[3] Giovannucci E, Liu Y, Hollis BW, et al. 25hydroxyvitamin D and risk of myocardial infarction in men: a prospective study. Arch Intern Med 2008;168(11):1174-80.
[4] Merke J, Milde P, Lewicka S, et al. Identification and regulation of 1,25-dihydroxyvitamin D3 receptor activity and biosynthesis of 1,25- dihydroxyvitamin D3. Studies in cultured bovine aortic endothelial cells and human dermal capillaries. J Clin Invest 1989;83(6):1903-15.

[5] Somjen D, Weisman Y, Kohen F, et al. 25Hydroxyvitamin D3-1 alpha-hydroxylase is expressed in human vascular smooth muscle cells and is up regulated by parathyroid hormone and estrogenic compounds. Circulation 2005;111(13):1666-71.

[6] Merke J, Hofmann W, Goldschmidt D, et al. Demonstration of 1,25 (OH)2 vitamin D3 receptors and actions in vascular smooth muscle cells in vitro. Calcif Tissue Int 1987;41(2):112-4.

[7] Holick MF. High prevalence of vitamin D inadequacy and implications for health. Mayo Clin Proc 2006;81(3):353-73.

[8] Wang TJ, Pencina MJ, Booth SL, et al. Vitamin D deficiency and risk of cardiovascular disease. Circulation 2008;117(4):503-11.

[9] Weishaar RE, Simpson RU. Vitamin D3 and cardiovascular function in rats. J Clin Invest 1987;79(6):1706-12.

[10] Sathyamurthy L, Shyam PK, Kirubakaran K, et al. Hydroxy vitamin D3 levels in acute coronary syndrome. Journal of Indian College of Cardiology 2012;2(4):141-3.

[11] Martins D, Wolf M, Pan D, et al. Prevalence of cardiovascular risk factors and the serum levels of 25hydroxyvitamin D in the United States: data from the Third National Health and Nutrition Examination Survey. Arch Intern Med 2007;167(11):1159-65.

[12] Pilz S, Tomaschitz A, Marz W, et al. Vitamin D, cardiovascular disease and mortality. Clin Endocrinol (Oxf) 2011;75(5):575-84.

[13] Hur SJ, Kim DM, Lim KH, et al. Vitamin D levels and their relationship with cardiac biomarkers in chronic Hemodialysis patients. J Korean Med Sci 2009;24(Suppl 1):S109-S14.

[14] Mahdavi K, Amirajam Z, Yazdankhah S, et al. The prevalence and prognostic role of vitamin D deficiency in patients with acute coronary syndrome: a single centre study in South-West of Iran. Heart Lung Circ 2013;22(5):346-51.

[15] Judd SE, Tangpricha V. Vitamin D deficiency and risk for cardiovascular disease. Am J Med Sci 2009;338(1):40-4. 